\title{
Bürgerzufriedenheit mit Portalen der öffentlichen Verwaltung - Ergebnisse einer Untersuchung über Zufriedenheitsmodelle und Vertrauensfragen im E-government
}

\author{
Ronald Roedl $^{1}$ and Mark Nadjafi ${ }^{2}$ \\ ${ }^{1}$ Amt der steiermärkischen Landesregierung. Referat für E-Government. \\ Burggasse 2, 8010 Graz, Austria \\ rroedl@maxwell.syr.edu \\ ${ }^{2}$ Amt der steiermärkischen Landesregierung. Referat für E-Government. \\ Burggasse 2, $8010 \mathrm{Graz}$, Austria \\ mark.nadjafi@stmk.gv.at
}

\begin{abstract}
Die vorliegende Arbeit beschäftigt sich mit der Messung der $\mathrm{Zu}$ friedenheit der Bürger/Nutzer von Portalen der öffentlichen Verwaltung bzw. worauf diese Zufriedenheit fußt und wie man im weiteren diese beeinflussen kann. Anhand den Ergebnissen einer Online Umfrage auf dem Online Portal des Land Steiermark wird ein Kausalmodell zu Messung der Bürgerzufriedenheit vorgestellt. Die Ergebnisse dieser Umfrage zeigten, dass es einen positiven Zusammenhang zwischen Zufriedenheit mit Informationsportalen und Vertrauen in öffentliche Institution bzw. der Informationsaufbereitung gibt.
\end{abstract}

\section{Einleitung}

In Hinblick auf den Einsatz und die geplante flächendeckende Ausweitung von EGovernment Service-Angeboten ist es unerlässlich sich mit der Frage zu beschäftigen, nach welchen Kriterien man die Zufriedenheit der Bürger mit dem Informationsangebot und im weiteren mit Leistungen messen kann, um damit eine geeignete und effektive Evaluierung vornehmen zu können. Der Erfolg von EGovernment wird letztlich davon abhängen, ob die Angebote den Bedürfnissen der Bürger entsprechen und auch von diesen tatsächlich genutzt werden.

In der wissenschaftlichen Literatur wurde dem Thema Zufriedenheit mit Websites der öffentlichen Verwaltung bis dato keine größere Aufmerksamkeit zuteil. Untersuchungen zum Thema Costumer-Satisfaction mit Websites oder Online-Portalen werden fast ausschließlich für E-Business Lösungen erarbeitet. Allerdings können aus den bisherigen Untersuchungen wertvolle Erkenntnisse gezogen werden. 


\section{Wissensmanagament im E-government}

E-Government ist in weiterem Sinne eine Form des Wissenmanagements, da durch die effektive Nutzung von Wissen, qualitativ zielführende Entscheidungen und eine höhere Effizienz in der Durchführung von Verwaltungsprozessen, erreicht werden kann. Das Sammeln von Wissen über die Bedürfnisse der Bürger, die als Kunden der öffentlichen Verwaltung anzusehen sind, kann dazu verwendet werden, einerseits die Akzeptanz der Angebote zu steigern und andererseits damit die Kosten für eine falsche Entwicklung und Umsetzung zu senken, was unmittelbar zu einer Qualitätssteigerung der Dienstleistung führt.

In Hinblick auf ein Wissensmanagement-System ist auch im E-Government der Wissenserwerb ein äußerst wichtiger Bestandteil, denn erst dadurch kann der Aufbau neues Wissens vonstatten gehen und der produktive Einsatz von Wissen zum Nutzen der Verwaltung garantiert werden. Daher sind die drei Säulen Wissenserwerb, Wissensentwicklung und Wissensnutzung die Fundamente für eine weitreichende Verbreitung des erworbenen Wissens, so dass die beiden Akteure, Bürger und Verwaltung, gleichsam davon profitieren können und damit ein Gleichgewicht zwischen Bedürfnisse und Machbarkeit herstellen. Insbesondere die Wissensnutzung stellt eine große Herausforderung dar, da bisher in der öffentlichen Verwaltung kaum bis gar nicht die Meinungen und Erfahrungen der Bürger berücksichtig wurden. EGovernment stellt daher in dieser Hinsicht eine Reform der Bürger-Verwaltungs Situation dar und fügt dem ein neues Element hinzu, so dass bei der Modernisierung der Verwaltung gleich die Bedürfnisse der Bürger integriert und berücksichtigt werden.

Im Gegensatz zum expliziten Wissen, welches kodifiziert oder jedenfalls formal artikulierbar, zum Beispiel in Form von grammatikalischen Aussagen, Spezifikationen oder Handbüchern existiert, ist das implizierte Wissen, also dass verborgene oder stillschweigende Wissen nicht leicht zugänglich. Das implizierte Wissen auch ,tacit knowledge" genannt, ist gebunden an das Individuum und nur schwer systematisch zu vermitteln, wie etwa der subjektive Einblick oder ein subjektives Verständnis eines Themas. Daher muss man, wenn man bestrebt ist Wissen über die Bedürfnisse der Bürger zu generieren, einen Weg finden um dieses implizierte Wissen aus der individuellen Perspektive in eine objektive Ebene herauszulösen und durch die Standardisierung und Objektivierung messbar zu machen.

Um das ,implizierte Wissen“ der Bürger zu bestimmten E-Government Leistungen zu erheben und dieses erhobene Wissen auch sinnvoll nutzen zu können, benötigt man spezielle Modelle, die auf Faktoren beruhen, mit deren Hilfe man Einstellungen wie Haltungen der Befragten eruieren und messen kann. In dieser Untersuchung wurde dafür das Bürgerzufriedenheitsmodell herangezogen.

\section{Bürgerzufriedenheitsmodelle}

Das Grundgerüst für das Bürgerzufriedenheitsmodell bildet das End-User-Computing Satisfaction Modell von Doll/Torkzadeh ${ }^{1}$, dass dazu dient um damit die Gesamtzufriedenheit der Anwender eines Systems zu eruieren. Schwerpunkt dieses Modells ist

\footnotetext{
${ }^{1}$ vgl. Doll/Torkzadeh, 1988
} 
die Untersuchung folgender Faktoren: Satisfaction, Ease of Use, Format, Content, Accuracy, Timeliness (siehe Abb. 2). Dieses Modell, das 1988 erarbeitet wurde, wurde mittlerweile von einer Vielzahl von Autoren in empirischen Untersuchungen bestätigt.

Chin/Lee $e^{2}$ erweiterten das Modell um Erwartungsfaktoren, damit wurden Verhaltenskomponenten in das Modell integriert. Andere Autoren haben dieses Modell für Untersuchungen bestimmter Systeme angewendet. Downing ${ }^{3}$, untersuchte die Zufriedenheit mit dem Produkt Savings Express und bestätigte dieses Modell als tauglich, Zufriedenheit mit einem Informationssystem zu messen. McHaney/Hightower/White 4 setzten dieses Modell ein, um die Zufriedenheit mit einem Decision Support System (DSS) zu untersuchen. Auch hier wurde bestätigt, dass das End-User Computing Satisfaction Modell ein hohes Maß an Varianz erklären kann. Wang/Tang/Tang ${ }^{5}$ erweiterten das EUCS Modell um diese branchenspezifischen Faktoren;“ Measures of user information satisfaction developed for the conventional data processing environment or end-user computing environment max no longer be appropriate for the digital marketing context, where the role of an individual costumer is in some ways different to that of an organizational end-user".

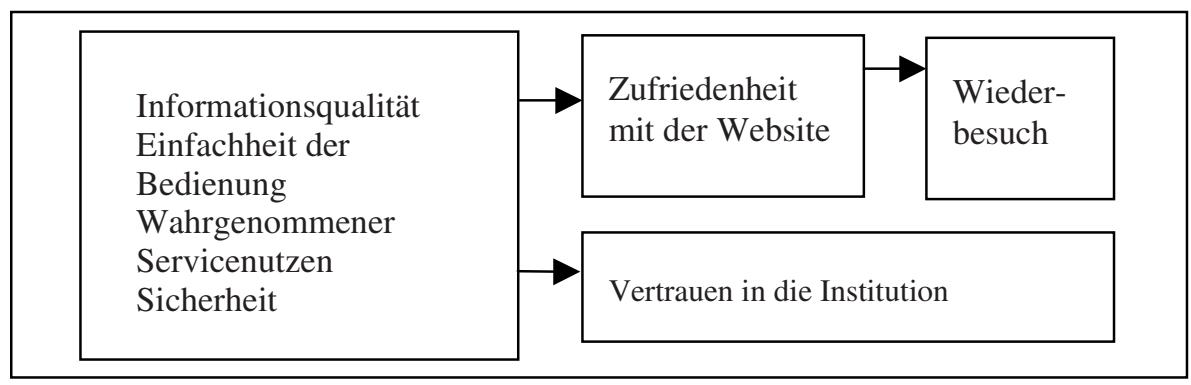

Fig. 1. Faktoren des BZM

Das End-User-Computing-Satisfaction Modell basiert auf folgende Faktoren: Satisfaction (Zufriedenheit der Endnutzer), Ease of Use (Benutzerfreundlichkeit des Systems), Format (Darstellung des Inhalts), Content (Aufbereitung des Inhalts), Accuracy (Fehlerfreiheit des Systems), Timeliness (Aktualität des Inhalts). Diese Faktoren ergeben die Gesamtzufriedenheit mit dem System und dienen als exogene Faktoren im EUCS, so dass sie als Grundlage der Operationalisierung der Erhebung herangezogen werden.

2 vgl. Chin, W.W.; Lee, M.: A Proposed Model for Measurement Instruments For The Formation of IS Satisfaction: The Case of End-User Computing Satisfaction, Proceedings of the Twenty First International Conference on Information Systems, 2000, S.553-563

3 vgl. Downing, 1998

4 vgl. McHaney, R.; Hightower, R.; White, D.: EUCS Test-Retest Reliability in Representational Model Decision Support Systems, Information and Management 36, 1999, S.109-119

5 vgl. Wang, Y-S.; Tang, T-I. ; Tang, J. E. : An Instrument for Measuring Costumer Satisfaction Toward Web Sites that Market Digital Products and Services, Journal of Electronic Commerce Research, Vol. 2, No. 3, 2001, S.93 


\begin{tabular}{|c|c|c|}
\hline EUCS & CIS & BZM \\
\hline Satisfaction & Satisfaction & Zufriedenheit \\
\hline Ease of Use & \multirow[b]{2}{*}{ Ease of Use } & \multirow[b]{2}{*}{$\begin{array}{c}\text { Einfachheit der } \\
\text { Bedienung }\end{array}$} \\
\hline Format & & \\
\hline Content & \multirow[b]{2}{*}{ Information Content } & \multirow{4}{*}{ Informationsqualität } \\
\hline Accuracy & & \\
\hline Timeliness & \multirow[b]{2}{*}{ Innovation } & \\
\hline & & \\
\hline & Security & Sicherheit \\
\hline & Products/Services & \multirow{3}{*}{ Servicenutzen } \\
\hline & Costumer Support & \\
\hline & Transaction and Payment & \\
\hline & & Vertrauen \\
\hline & & Wiederbesuch \\
\hline
\end{tabular}

Fig. 2. Vergleich zwischen EUCS, CIS und BZM

Das Bürgerzufriedenheitsmodell BMZ, welches als Basis für die Hypothesen dieser Arbeit herangezogen wird und eine Ableitung des EUCS ist, soll jene Erkenntnisse, die im E-Business erlangt wurden auf die öffentliche Verwaltung übertragen. Dieses Modell nutzt jene Determinanten, die für eine Zufriedenheitsanalyse von Websites der öffentlichen Verwaltung Geltung haben und ergänzt jene branchenspezifischen Determinanten, die bisher kaum Beachtung fanden. Von großem Interesse ist die Einbeziehung des Faktors Vertrauen.

Wie in Fig.1 ersichtlich, beeinflussen die exogenen Faktoren Informationsqualität, Einfachheit der Bedienung (sprich Usability), wahrgenommener Service-Nutzen und Sicherheit die endogenen Faktoren Zufriedenheit der Website und Vertrauen in die Institution, das wiederum einen Einfluss auf den Wiederbesuch ausübt.

Im folgenden wird ein Vergleich der exogenen Konstrukte des End-User Computing Satisfaction Modells (EUCS), des Costumer Information Satisfaction Modells (CIS), welches ein ebenso abgeleitetes Modell ist, und des Bürgerzufriedenheitsmodells (BZM) gezeigt.

Wie aus der Fig. 2 ersichtlich, werden bei den Modellen EUCS und CIS die Faktoren Vertrauen und Wiederbesuch nicht berücksichtigt. Das BZM bezieht sich in seiner Modellgestaltung direkt auf jene Faktoren, die insbesondere für E-Government vordergründig sind, deshalb werden diese spezifischen Faktoren, die beim EUCS nicht berücksichtigt werden, hier kurz skizziert. 


\subsection{Vertrauen}

In der Business-to-Consumer Forschung (B2C) wurde in den aktuellsten Studien das Thema Vertrauen (Trust), der Ruf eines Unternehmens (Reputation), Glaubwürdigkeit (Credibility) und Vertrauenswürdigkeit in ein Unternehmen (Trustworthiness) große Beachtung zuteil. Sultan/Urban/Shankar/Bart ${ }^{6}$ betonen die Notwendigkeit der Untersuchung dieser Determinanten, "Trust is important and understanding how it works in the online environment is essential for firms to develop a long-term relationship with consumers."

Pavlou zeigt in seiner Untersuchung7, dass Trust einen erheblichen Einfluss auf das Verhalten von Online-Konsumenten hat," Trust influences online transaction intentions, inferring that this behavorial and control belief is important for e-commerce adoption.“. Ba/Pavlou 8 stellen fest, dass die Reputation eines Unternehmens einen Einfluss auf die weiteren Absichten eines Käufers im Internet hat "Our hypotheses are largely supported and suggest that a seller`s reputation, reflected in his feedback profile, plays a very important role in buyers ' willingness to pay premium prices". Fogg/Kameda et.al und Fogg/Marshall et.al. ${ }^{9}$ befassten sich mit dem Thema "Credibility" 10 von Websites und stellten unter anderem fest, dass das Unternehmen, wie es von Usern wahrgenommen wird, Einfluss auf die Zufriedenheit mit der Website hat:" An organization's standing in the real world impacts the credibility of its institutional website. Not surprisingly, the results say that "well respected" companies also enjoy high levels of online credibility, while companies with legal or financial troubles garner low rankings."

Koufaris/Hampton-Sousa ${ }^{11}$ bestätigten einen Zusammenhang zwischen der Vertrauenswürdigkeit in ein Unternehmen und der Kaufabsicht eines Users. Die wahrgenommene Vertrauenswürdigkeit des Unternehmens, der Organisation hat also Einfluss auf die Zufriedenheit mit einer Website, bzw. Einfluss auf weitere Verhaltensabsichten des Users. Das Konstrukt „Vertrauenswürdigkeit des Unternehmens“, findet für das Bürgerzufriedenheitsmodell Verwendung als Konstrukt „Vertrauenswürdig-

6 vgl. Sultan, F.; Urban, G.L., ShankarR, V., Bart, I.Y.: Determinants and Role of Trust in EBusiness: A Large Empirical Study, eBusiness Research center Working Paper, Dec. 2002, S.6

7 vgl. Pavlou, P. A. : Consumer Intentions to Adopt Electronic Commerce - Incorporating Trust and Risk in the Technology Acceptance Model, Paper to be presented in the Dgit Workshop, 2001, 1-28; BA, S.; Pavlou, P.A.: Evidence of the Effect of Trust Building Technology in Electronic Markets: Price Premiums and Buyer Behavior, MIS Quarterly, Vol.26, No.3, Sep. 2002, S. 243-268

8 vgl. Ba/Pavlou, 2002, S.261

9 vgl. Fogg, B.J; Kameda, T; Boyd, J.; Marshall, J.; Sethi, R.; Sockol, M.; Trowebridge, T.: Stanford Makovsky Credibility Study 2002: Investigating what makes Web sites credible today, Research Report by the Stanford Persuasive Technology Lab \& Makovsky \& Company, 2002, S.1-16; Fogg, B.J; Marshall, J.; Laraki, O.; et al.: What makes Web Sites Credible?, Report on a large Quantitative Study, Persuasive Technology Lab-Chi-letters, Stanford University, 2001, S.61-68

${ }^{10}$ vgl. Fogg/Marshall,/Laraki et al., 2002, S.14

${ }^{11}$ vgl. Koufaris, M.; Hampton-Sosa, W. :Initial Perceptions of Company Trustworthiness Online : A Comprehensive Model and Empirical Test, CIS Working Paper Series, 2002, S.1-39 
keit der Institution“. Diese Erkenntnisse werden in bezug auf die öffentliche Verwaltung untersucht.

\subsection{Zufriedenheit}

Die Zufriedenheit mit einer Website wird generell als ein wichtiger Faktor des Erfolgs von Informationstechnologie angesehen ${ }^{12}$. In der öffentlichen Verwaltung gibt es bis zum heutigen Tag keine Instrumente, die angewendet werden, um die Zufriedenheit mit einer angebotenen Website bzw. Informationsportal zu untersuchen. Untersucht werden soll eine generelle Zufriedenheit bzw. die Zufriedenheit mit dem persönlichen Erfolg auf der Website

\subsection{Wiederbesuch}

Die Frage nach der Nutzung der Website steht im Vordergrund der empirischen Untersuchung. Die Wiederbesuchsabsicht und der tatsächliche Wiederbesuch sind für den Website Betreiber wichtige Indikatoren für die Erfolgsmessung.

\section{Informationsportale der öffentlichen Verwaltung}

Obwohl Bürger die Bedienung am Schalter im allgemeinen als eher freundlich erleben, gibt es doch eine tiefverwurzelte Abneigung, überhaupt mit der Verwaltung in Kontakt zu treten. Umso verständlicher ist es, dass ein hoher Prozentsatz der Bevölkerung sowohl die typischen Hindernisleistungen als auch die Kontaktaufnahme zu Stellen der Verwaltung künftig Online erledigen will. Über einheitliche Portale kann der Zugang von Bürgern zu allen Leistungen erheblich verbessert werden. Andererseits schafft die Vielfalt an angebotenen Leistungen im Behördendickicht auch Probleme. Ohne Orientierungsunterstützung ${ }^{13}$ kann die Vielfalt an Informations-, Kommunikations-, Produkt- und Verfahrensangeboten im Internet allenfalls zufällig oder

nach Lernen aus längerer eigener Erfahrung genutzt werden. Portale hingegen bündeln diese Informations- und Kommunikationsangebote und ermöglichen eine verbesserte und übersichtlichere Orientierung für die Nutzer.

Lucke $^{14}$ definiert ein Portal als ein Eingangstor zu einer virtuellen Gemeinschaft ${ }^{15}$. Dieses Portal eröffnet dem Besucher den Eintritt in ein bestimmtes Themengebiet, das durch dieses Portal geöffnet und erschlossen wird. Portale sind in der Regel um be-

\footnotetext{
12 vgl. Xiao/Dasgupta, 2002, S.1149

${ }^{13}$ vgl. Reinermann, H.: Portale - Tore des Wissens, in: Reinermann, H.; Lucke, J. von: Portale in der öffentlichen Verwaltung, Internet - Call Center - Bürgerbüro, Speyerer Forschungsbericht Nr. 205, Speyer, 2000, S.1

${ }^{14}$ vgl. Lucke, J. von: Portale für die öffentliche Verwaltung, Governmental Portal, Departmental Portal und Life-Event Portal, in: Reinermann/Lucke, 2000, S.7

${ }^{15}$ Community
} 
stimmte Regionen (Regional Portal), um bestimmte Themen (Themen-Portal) oder um bestimmte Lebensphasen (Life-Event Portal) angelegt.

Ein Portal stellt nichts anderes als eine Website dar, die übersichtlich nach Inhalten strukturiert ist und ausgewählte Inhalte des World Wide Web (WWW) redaktionell aufarbeitet. Anwender nutzen Portale gerne, weil sie kostenlos, schnell und übersichtlich $\mathrm{zu}$ gewünschten Angeboten führen und vielfach noch nützliche Mehrwertdienste $^{16}$ besitzen.

Innovative und fortschrittliche Verwaltungen planen ihren Internetauftritt nach dem Portalkonzept ${ }^{17}$, Onlinedienste werden nicht mehr nach dem Behördenkonzept, sondern behördenübergreifend erarbeitet. Grundsätzlich lassen sich drei Typen von Portalen in der öffentlichen Verwaltung unterscheiden: Portale, die sich um Gebietskörperschaften gruppieren (Governmental Portal), um Ämter, Behörden und Ministerien (Departmental Portal) oder um bestimmte Aufgaben und Lebensphasen (LifeEvent Portal).

Unter dem Begriff „Governmental Portal“ 18 sind die Portale der Gebietskörperschaften (in Österreich: Bund, Länder und Gemeinden) zusammenzufassen. Sie verweisen in der Regel auf die elektronischen Angebote ${ }^{19}$ der ihnen zugehörigen Ministerien, Behörden und sonstigen Einrichtungen. Häufig bestehen Verknüpfungen zu den elektronischen Angeboten der über- und untergeordneten Verwaltungseinheiten.

Der Begriff „Departmental Portal“ ${ }^{\circ 2}$ umschließt alle Portale, die im Hinblick auf eine bestimmte Verwaltungseinheit (Ministerium, Behörde oder Amt) gebildet wurden. In der Regel handelt es sich um die Website ${ }^{21}$ der Verwaltungseinheit. Für diese Form ist es typisch, dass jede Verwaltungseinheit über eine eigene Homepage, ein eigenes Angebot und eigene Transaktionslösungen verfügt.

Sowohl das Governmental Portal als auch das Departmental Portal haben den entscheidenden Nachteil, dass sich der Bürger über die Struktur und die Geschäftsverteilung der Verwaltung informieren muss.

\section{Ergebnisse der Online-Befragung auf dem Departmental Portal ,Land Steiermark“6}

Die Online-Umfrage auf dem Portal Land Steiermark (http://www.steiermark.at) hatte zum Ziel die Zufriedenheit der Nutzer mit dem Informationsangebot, der Usability und dem subjektiven Empfinden des Service-Nutzen zu erheben. Durch das Erfragen von persönlichen Daten sollten die derzeitigen Nutzertypen und deren Internetaffinität bzw. die Besuchshäufigkeit identifiziert und im weiteren das typische Nutzerprofil abgeleitet werden.

\footnotetext{
16 vgl. Lucke, 2000, S.7

${ }^{17}$ vgl. Caldow, J.: The Quest for Electronic Government - A Defining Vision, Institute for Electronic Government, IBM Corporation, Washington, 1999, S.7

http://www.ieg.ibm.com/thought_leadership/egovvision.pdf (12.5.2003)

18 vgl. Lucke, 2000, S.13

${ }^{19}$ Als Beispiele: http://www.austria.gv.at (19.3.2003), http://www.stmk.gv.at (19.3.2003)

${ }^{20}$ vgl. Lucke, 2000, S.13

${ }^{21}$ Als Beispiele: http://www.bmaa.gv.at (19.3.2003), http://www.fa1a.gv.at (20.3.2003)
} 
Neben diesen Aspekten sollte die Umfrage Aufschluss darüber bringen, was sich die Nutzer von einem guten Internetauftritt generell erwarten und wie sie die Sicherheitsaspekte des Steiermark Portals beurteilen. Es wurde konkret nach der Einschätzung folgender Aspekte gefragt:

- Bedienung der Website

- Information

- Vertrauen

- Sicherheit

- Serviceleistung

- Zufriedenheit

- Wiederbesuchsabsicht

Durch die Ergebnisse der Umfrage sollten sinnvolle Handlungsempfehlungen für eine Optimierung der Website erstellt werden.

Die Umfrage lief 8 Tage. Das Pop- up Fenster wurde 15283 mal aufgerufen. Ingesamt haben 572 Personen die Befragung korrekt und vollständig ausgefüllt. 800 Personen haben die Befragung abgebrochen. 61,7\% haben sich für das Gewinnspiel mit Anschrift und Name registriert.

\subsection{Ergebnisse der Umfrage}

\section{Generelle Häufigkeiten}

Auf die Frage nach dem Wiederbesuch der Website gaben 48,2 \% der Befragten an, dass sie dies sicher wieder tun werden und 38,7 \% gaben an, dass sie dies eher tun werden. Die Mehrheit der Befragten ist einem Wiederbesuch der Website positiv eingestellt.

Mit Hilfe der Frage nach dem Vertrauensverhältnis sollte erhoben werden, wie weit die Befragten die Institution Land Steiermark vertrauen und als vertrauenswürdigen Partner empfinden bzw. ob die Auskünfte und Informationen als glaubwürdig empfunden wurden.

$52,1 \%$ stimmten der Frage ob das Land Steiermark ein vertrauenswürdiger Partner sei voll zu, 28,0 \% stimmten diesen eher zu. Der Großteil der Befragten besitzt ein großes Vertrauen in die Institution Land Steiermark. Siehe dazu auch Fig. 3.

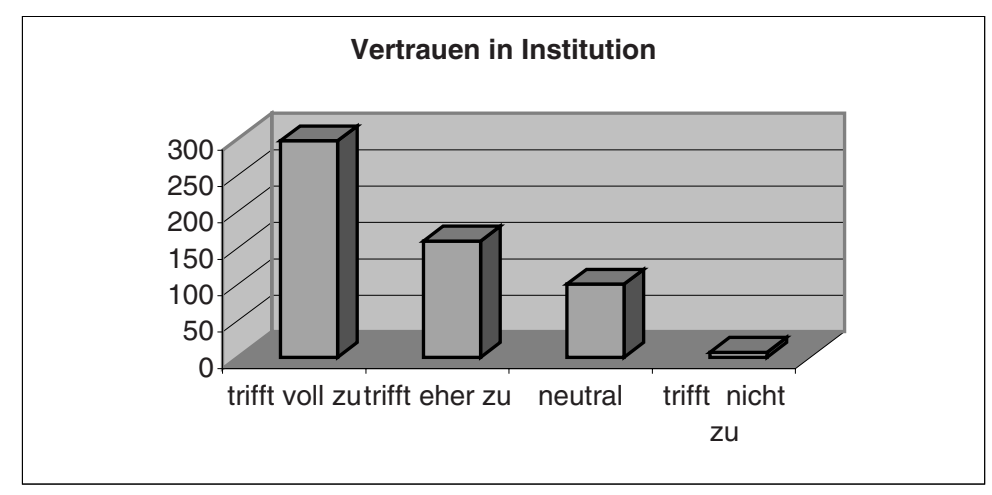

Fig. 3. Häufigkeit der Beurteilung, ob das Land Steiermark ein vertrauenswürdiger Partner ist. 
Das Land Steiermark wird außerdem von der Mehrheit als eine verlässliche Institution eingestuft. 48, $1 \%$ haben dem voll zu gestimmt und 30, $2 \%$ stimmten dem eher zu. Allerdings haben auch 19,2 \% der Befragten eine neutrale Haltung zu dieser Frage.

Die Glaubwürdigkeit der Auskünfte und Informationen des Landes Steiermark werden sehr hoch zugestimmt. 50,3\% der Befragten stimmen dem voll zu und 29, 2 $\%$ stimmen dem eher zu. Die Befragten sind demnach von der Glaubwürdigkeit der Information großteils überzeugt.

In bezug auf die allgemeine Zufriedenheit mit der Website sind $28,1 \%$ völlig zufrieden und 47,5\% eher zufrieden. 19,7\% besitzen eine neutrale Meinung dazu. In dieser Hinsicht gibt es daher sicherlich noch ein Handlungsbedarf zu einer Optimierung der Website.

Das Maß an Zufriedenheit mit dem Service-Angebot der Website wird von 49, $7 \%$ der Befragten als ,stimme eher zu“ beurteilt und von 21, $5 \%$ als neutral. Völlig zufrieden sind 22, 7\% der Befragten. Die Zufriedenheit mit dem Service-Angebot liegt daher im positiven Mittelfeld.

Bei dem Vertrauen in der Datenübermittlung ist eine ähnliche Haltung festzustellen. 66, $6 \%$ beurteilen diese im mittleren Feld der Skala und sind somit nicht völlig von der Sicherheit des Transfers von Daten überzeugt. Nur 29, 3 \% sind völlig davon überzeugt. Allerdings entspricht dies der generellen Skepsis der Internet-Nutzer über die Sicherheit der Datenübermittlung, wie auch nationale und internationale Studien zeigen.

\section{2 Überprüfung der Zusammenhänge zwischen den Variablen}

Die Daten wurden im Rahmen einer Faktorenanalyse, der Analyse des Cronbachschen Alphas und der Item-to-Total Korrelation auf Zusammenhänge überprüft. Die deskriptiven Auswertungen erfolgten mittels Errechnung von Häufigkeiten und Kreuztabellen.

\subsubsection{Faktorenanalyse}

Die Eignung der vorhandenen Datengrundlage für eine Faktorenanalyse konnte mittels der Prüfung des Kaiser-Meyer-Olkin Kriteriums nachgewiesen werden. Das Kaiser-Meyer-Olkin Kriterium zeigt an, in welchem Umfang die Ausgangsvariablen zusammengehören und dient somit als Indikator dafür, ob eine Faktorenanalyse sinnvoll erscheint oder nicht. Kaiser/Rice ${ }^{22}$ vertreten die Meinung, dass sich ein Wert unter 0,5 nicht für eine Faktorenanalyse eignet, Werte über 0,7 werden als ziemlich gut, Werte über 0,8 als verdienstvoll eingestuft.

Für die exogenen Variablen wurde ein Wert von 0,825 erzielt, für die endogenen Variablen ein Wert von 0,826. Daher kann eine Faktorenanalyse durchgeführt werden. Die MSA-Kriterien (measure of sampling adequacy) für die einzelnen Variablen liegen bei allen Variablen über 0,7, was als zumindest ,ziemlich gut“ beurteilt werden kann.

${ }^{22}$ vgl. Kaiser, H.F.; Rice, J.: Little Jiffy, Mark IV, in: Educational and Psychological Measurement, 34, 1974, S.111f 


\begin{tabular}{|c|c|c|c|c|c|}
\hline Faktor & Variable & Bezeichnung & Faktorladung & MSA & Varianz \\
\hline Information & $\mathrm{x}_{1}$ & Verständlichkeit & 0,80 & 0,93 & \multirow[t]{2}{*}{$19,43 \%$} \\
\hline & $\mathrm{x}_{2}$ & Bedürfniserfüllung & 0,74 & 0,93 & \\
\hline $\begin{array}{l}\text { Einfachheit der } \\
\text { Bedienung }\end{array}$ & $\mathrm{x}_{1}$ & $\begin{array}{l}\text { Anwender- } \\
\text { freundlichkeit }\end{array}$ & 0,86 & 0,83 & \multirow[t]{2}{*}{$22,73 \%$} \\
\hline & $\overline{x_{3}}$ & Bedienbarkeit & 0,85 & 0,83 & \\
\hline \multirow{2}{*}{$\begin{array}{l}\text { Wahrgenommener } \\
\text { Servicenutzen }\end{array}$} & $\mathrm{x}_{8}$ & Serviceangebot & 0,84 & 0,83 & \multirow[t]{2}{*}{$22,91 \%$} \\
\hline & $\mathrm{X}_{9}$ & Bedarfsorientierung & 0,85 & 0,82 & \\
\hline \multirow[t]{3}{*}{ Sicherheit } & $\mathrm{x}_{6}$ & Sicherheit der Website & 0,91 & 0,71 & \multirow[t]{2}{*}{$23,22 \%$} \\
\hline & $\mathrm{x}_{7}$ & Datenübermittlung & 0,92 & 0,71 & \\
\hline & & \multicolumn{2}{|l|}{$\begin{array}{l}\text { KMO: } 0,825 \\
\text { Signifikanzniveau: } 0,000\end{array}$} & & $\begin{array}{l}\text { Summe: } \\
88,29 \%\end{array}$ \\
\hline
\end{tabular}

Fig. 4. Faktorenanalyse exogene Faktoren

\begin{tabular}{|c|c|c|c|c|c|}
\hline Faktor & Variable & Bezeichnung & Faktorladung & MSA & Varianz \\
\hline \multirow[t]{3}{*}{$\begin{array}{l}\text { Vertrauen in } \\
\text { Institution }\end{array}$} & $\mathrm{y}_{1}$ & Vertrauenswürdigkeit & 0,91 & 0,78 & \multirow[t]{3}{*}{$37,58 \%$} \\
\hline & $\mathrm{y}_{2}$ & $\begin{array}{l}\text { Glaubwürdigkeit der } \\
\text { Institution }\end{array}$ & 0,86 & 0,92 & \\
\hline & $\mathrm{y}_{3}$ & $\begin{array}{l}\text { Verlässlichkeit d. } \\
\text { Institution }\end{array}$ & 0,92 & 0,77 & \\
\hline \multirow[t]{2}{*}{ Zufriedenheit } & $\mathrm{y}_{4}$ & Allg. Zufriedenheit & 0,85 & 0,84 & \multirow[t]{2}{*}{$24,55 \%$} \\
\hline & $y_{5}$ & Zielerreichung & 0,84 & 0,82 & \\
\hline \multirow[t]{3}{*}{ Wiederbesuch } & $y_{6}$ & Wiederbesuchsabsicht & 0,77 & 0,84 & \multirow[t]{2}{*}{$23,52 \%$} \\
\hline & $\mathrm{y}_{7}$ & Wiederbesuch & 0,88 & 0,84 & \\
\hline & & \multicolumn{2}{|l|}{$\begin{array}{l}\text { KMO: } 0,826 \\
\text { Signifikanzniveau } 0,000\end{array}$} & & $\begin{array}{l}\text { Summe: } \\
85,66 \%\end{array}$ \\
\hline
\end{tabular}

Fig. 5. Faktorenanalyse endogene Faktoren

Die Faktorladungen, die in der Faktorenanalyse erhoben wurden, übersteigen den geforderten Mindestwert ${ }^{23}$ von mindestens 0,5. Aus Fig. 4 und Abb. 5 ist zu entnehmen, dass alle Variablen den Mindestwert deutlich übertreffen.

Für die Bestimmung der Güte der Faktorenlösung ist die Frage der erklärten Varianz der Faktoren von Wichtigkeit. Die in Abb. 4 ausgewiesenen Varianzerklärungsanteile geben an, wie viel der jeweilige Faktor an Erklärungsanteil in bezug auf alle Ausgangsvariablen besitzt ${ }^{24}$.

Die vier exogenen Faktoren erklären 88,29\% der Ausgangsvarianz, wobei der erste Faktor $19,43 \%$, der zweite Faktor 22,73\%, der dritte Faktor 22,91\% und der vierte Faktor 23,22\% erklärt. Die endogenen Faktoren erklären zusammen 85,66\% der Ausgangsvarianz, wobei der erste Faktor 37,58\%, der zweite Faktor 24,55\% und der dritte Faktor $23,52 \%$ erklärt.

${ }^{23}$ vgl. Backhaus et al., 2003, S. 331

${ }^{24}$ vgl. Backhaus et al., 2003, S. 316 


\subsection{3 Überprüfung der Modellhypothesen}

Die kausalanalytische Modellprüfung ermöglicht direkte und indirekte Effekte des Modells zu analysieren. Aus den Koeffizienten des Strukturmodells ist zu entnehmen, dass $91 \%$ der Varianz des Konstrukts Zufriedenheit, 38\% der Varianz des Konstrukts Vertrauen in die Institution und 51\% der Varianz des Faktors Wiederbesuch erklärt werden können. Diese Werte werden aus Fig. 6 ,Squared Multiple Correlation" der AMOS-Auswertung entnommen. Vor allem das Konstrukt Zufriedenheit sticht mit einem hohen Wert von $91 \%$ hervor und bestätigt die Annahme, dass alle wesentlichen Variablen die Zufriedenheit erklären, gefunden werden konnten ${ }^{25}$.

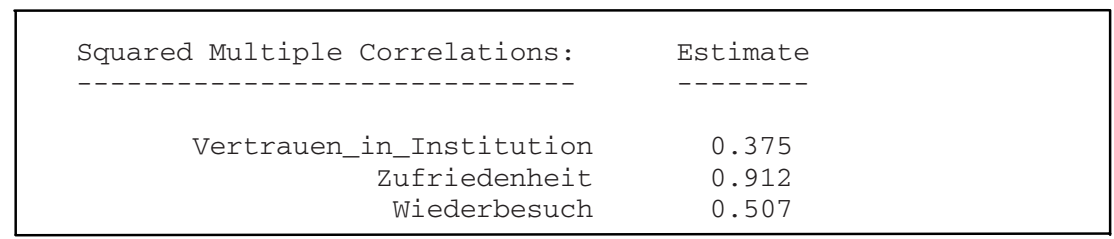

Fig. 6. Quadrierte multiple Korrelationskoeffizienten (Auszug aus AMOS-Analyse)

Die direkten Effekte, die von den exogenen Konstrukten auf die endogenen Konstrukte wirken, lassen sich aus Fig.7 entnehmen. Am stärksten wird die Zufriedenheit vom wahrgenommenen Servicenutzen mit 0,48 beeinflusst. Damit erfolgt eine Bestätigung von $\mathrm{H} 3$ (Wahrgenommener Servicenutzen $\rightarrow$ Zufriedenheit).

Ein hoher Zusammenhang besteht mit der Einfachheit der Bedienung mit 0,28. Damit kann $\mathrm{H} 2$ ebenso bestätigt werden (Einfachheit der Bedienung $\rightarrow$ Zufriedenheit).

Es ist ein Zusammenhang zwischen der Informationsqualität und dem Vertrauen in die Institution mit 0,21 nachweisbar, was H5 bestätigt (Informationsqualität $\rightarrow$ Vertrauen in die Institution).

Ein hoher Zusammenhang besteht ebenso zwischen der Sicherheit und dem Vertrauen in die Institution mit 0,48. Damit wird H6 bestätigt (Sicherheit $\rightarrow$ Vertrauen in die Institution).

Es ist auch ein deutlicher Zusammenhang zwischen dem Vertrauen in die Institution und der Zufriedenheit mit 0,12 nachweisbar. Damit wird H7 bestätigt (Vertrauen in die Institution $\rightarrow$ Zufriedenheit).

\begin{tabular}{|lc|}
\hline Standardized Regression Weights: & Estimate \\
- & \\
& \\
Vertrauen_in_Instituti <- Sicherheit & 0.475 \\
Vertrauen_in_Ins <- Informations_qua & 0.215 \\
Zufriedenheit <- Wahrgenommener_Serv & 0.482 \\
Zufriedenheit <- Einfachheit_der_Bed & 0.276 \\
Zufriedenheit <- Vertrauen_in_Instit & 0.117 \\
Zufriedenheit <--------_Sicherheit & 0.079 \\
Zufriedenheit <- Informations_qualit & 0.167 \\
Wiederbesuch <-------- Zufriedenheit & 0.532 \\
Wiederbesuch <- Vertrauen_in_Institu & 0.269 \\
\hline
\end{tabular}

Fig. 7. Direkte Effekte auf endogene Konstrukte (Auszug aus AMOS-Analyse)

${ }^{25}$ vgl. Backhaus et al., 2003, S.399 
Die Sicherheit spielt ebenso eine Rolle mit 0,08, was zu einer Bestätigung von H4 führt (Sicherheit $\rightarrow$ Zufriedenheit). Auch hier gibt es einen indirekten Beeinflussungseffekt $(0,439 * 0,125)$, der zu einer Gesamtbeeinflussung von 0,13 führt und damit einen höheren Wert als das Vertrauen in die Institution alleine erreicht.

Die Informationsqualität beschreibt einen mittleren Zusammenhang mit 0,17, womit eine Bestätigung von $\mathrm{H} 1$ folgt (Informationsqualität $\rightarrow$ Zufriedenheit). Hier kommt aber auch ein indirekter Beeinflussungseffekt zum Tragen, da die Informationsqualität auch über das Konstrukt Vertrauen auf die Zufriedenheit einwirkt. Der indirekte Effekt errechnet sich aus der unstandardisierten Lösung aus der Tabelle „Regression Weights“ $\left(0,233^{*} 0,125\right)$ und ergibt daher einen Gesamteffekt von 0,22.

Die Zufriedenheit wirkt auf den Wiederbesuch mit 0,53, dies bestätigt H8 (Zufriedenheit $\rightarrow$ Wiederbesuch)

Das Vertrauen in die Institution beeinflusst den Wiederbesuch mit 0,27; was H9 bestätigt (Vertrauen in die Institution $\rightarrow$ Wiederbesuch). Auch hier gibt es einen indirekten Beeinflussungseffekt $(0,125 * 0,48)$, der zu einer Gesamtbeeinflussung von 0,32 führt.

Aus der standardisierten Lösung kann man die Zusammenhänge zwischen den exogenen Konstrukten entnehmen. Hier zeigt sich, dass der höchste Zusammenhang zwischen der Informationsqualität und dem wahrgenommenen Servicenutzen mit 0,84 besteht. Die Informationsqualität korreliert auch mit 0,79 sehr hoch mit der Einfachheit der Bedienung, mit 0,51 geringer mit der Sicherheit der Website. Zwischen der Einfachheit der Bedienung und dem Servicenutzen gibt es eine hohe Korrelation mit 0,69; zwischen Einfachheit der Bedienung und der Sicherheit ist ein niedrigerer Wert - 0,4 - ablesbar. Zwischen dem Servicenutzen und der Sicherheit der Website besteht mit 0,46 ebenfalls ein Zusammenhang. Das Gesamtmodell kann daher folgendermaßen abgebildet werden (siehe Fig. 8).

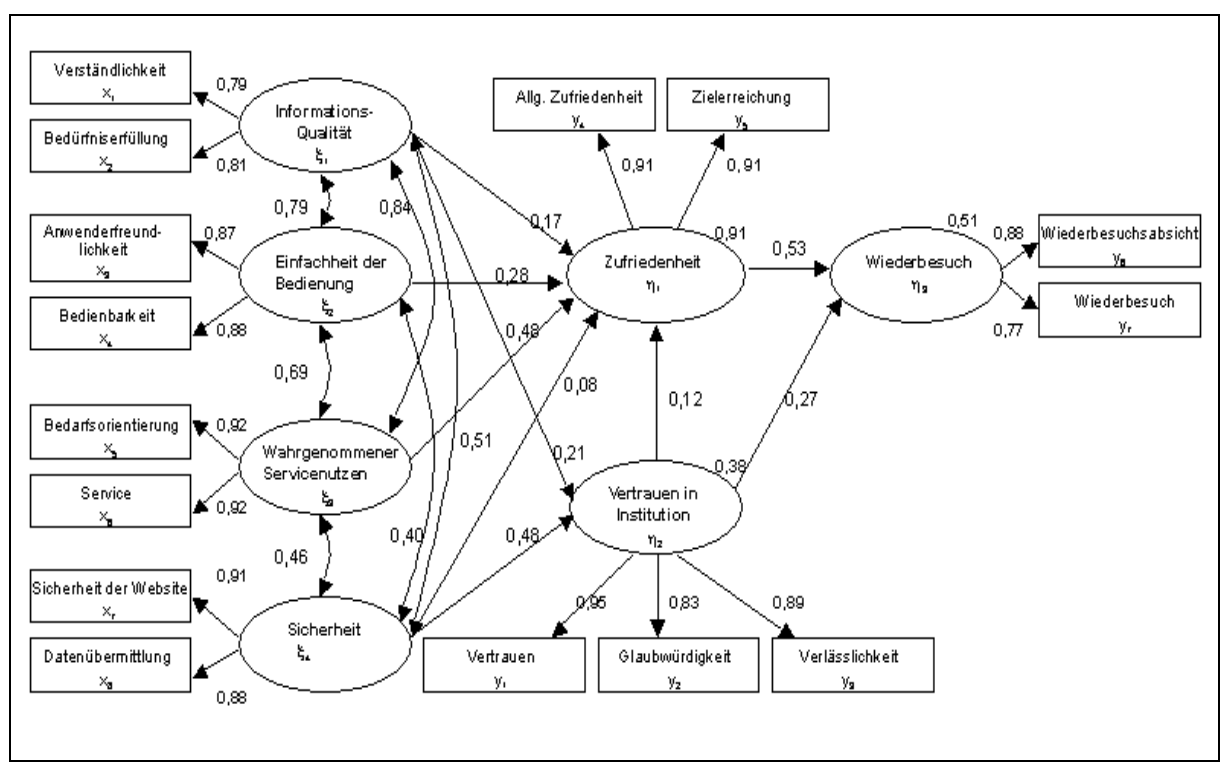

Fig. 8. Gesamtabbildung 


\section{Schlussfolgerung und Handlungsempfehlungen}

Überraschend ist bei der Analyse der Ergebnisse, dass das Vertrauen in die Institution öffentliche Verwaltung von hoher Bedeutung ist. Das Vertrauen in die Institution wird ja vorwiegend in der „Offline Welt“ gebildet, nimmt aber im Rahmen der Nutzung großen Einfluss auf den Wiederbesuch der Website. Dies ist insoweit bemerkenswert, als da nachgewiesen wurde, dass systemunabhängige Faktoren einen erheblichen Einfluss auf das Nutzerverhalten ausüben.

Die Einfachheit der Bedienung einer Website übt auch einen hohen Einfluss auf die Zufriedenheit der User mit der Website aus. Dies entspricht den Erkenntnissen, die ähnliche Fragestellungen über das Nutzungsverhalten bei Nutzern von EBusiness Websites ergeben haben. Nach dem wahrgenommenen Servicenutzen ist die Einfachheit der Bedienung der zweitwichtigste systemabhängige Faktor zur Erklärung der Nutzungszufriedenheit.

Die wahrgenommene Informationsqualität ist jener weitere Faktor, der den Erkenntnissen aus vergleichbaren E-Business Untersuchungen entspricht und einen Einfluss auf die Zufriedenheit mit der Website beschreibt. Es konnte auch nachgewiesen werden, dass der wahrgenommene Servicenutzen einen wichtigeren Stellenwert als die wahrgenommene Informationsqualität einnimmt. Die Erklärung dafür ist im Wandel der Ausrichtung von Websites der öffentlichen Verwaltung von Informations- zu Transaktionsportalen zu finden.

Die Sicherheit ist nur im Zusammenhang mit dem Vertrauen in die Institution von Bedeutung. Eine positive wahrgenommene Sicherheit einer Website hat direkt zwar geringen Einfluss auf die Zufriedenheit, beeinflusst aber in hohem Masse das Vertrauen in die Institution.

\subsection{Die Ausrichtung auf den Servicenutzen als höchste Priorität}

Der wahrgenommenen Servicenutzen steht für die Nutzer von Websites der öffentlichen Verwaltung im Vordergrund. Werden Services nicht angeboten, bzw. werden die falschen Services angeboten, d.h. Services, die sich nicht am Bedarf der Nutzer orientieren, hat dies erheblichen Einfluss auf die Zufriedenheit der Nutzer.

Öffentliche Verwaltungen sind daher in zweifacher Richtung gefordert: Erstens, Websites müssen auf das Vorhandensein von Services und Dienstleistungen überprüft werden, da reine Informationsseiten - die in der öffentlichen Verwaltung noch in großer Zahl vorhanden sind - nicht mehr die gewünschte Wirkung erzielen.

Zweitens, bestehende Services müssen auf ihre Bedarfsorientierung hin evaluiert werden, gegebenenfalls vom Netz genommen werden, in den meisten Fällen aber erst neu aufgebaut werden. Das heißt konkret, Verwaltungen können sich EGovernment Services nicht entziehen, wollen sie bürgerorientiert bleiben und eine hohe Nutzerzufriedenheit erlangen. 


\subsection{Vertrauen schaffen durch eine bürgerorientierte E-government Corporate Identity}

Wie oben dargestellt, hat das Vertrauen in die Institution einen deutlichen Einfluss auf die Zufriedenheit und vor allem einen erheblichen Einfluss auf den Wiederbesuch einer Website der öffentlichen Verwaltung. Das Vertrauen wird aber im direkten Kontakt mit Behörden, mit Mitarbeitern der öffentlichen Verwaltung und durch persönlichen Erfahrungen gebildet.

Wenn das Vertrauen in die Verwaltung hoch ist, hat dies Einfluss auf die Zufriedenheit mit der besuchten Website. Eine Corporate Identity, die Vertrauen vermittelt und E-Government als wünschenswert für den Bürger und die Verwaltung darstellt, schafft Vertrauen und damit höhere Zufriedenheit für Nutzer von Websites der öffentlichen Verwaltung. Dieses Vertrauen wird dann noch weiter verstärkt, wenn die Sicherheitselemente einer Website kommuniziert wird und wenn von Seiten der Verwaltung klargestellt wird, was mit übermittelten Daten im backoffice Bereich der Verwaltung geschieht. Daher macht es Sinn, wenn auf Websites der öffentlichen Verwaltung dem Thema der Datensicherheit und der Website-Sicherheit größere Bedeutung zugemessen wird.

Die Corporate Identity Verantwortlichen einer Verwaltung, aber auch das oberste Verwaltungsmanagement und die politischen Verantwortungsträger müssen sich der Tatsache bewusst sein (und im besten Fall ihr Handeln danach ausrichten), dass ein Verlust an Vertrauen in die Verwaltung auch bei einer noch so gut gestalteten Website zu negativen Auswirkungen bei der Zufriedenheit der Nutzer dieser Website führt.

\subsection{Usability-Orientierung und Informationsarchitektur als Basis von erfolgreichen Verwaltungs-Websites}

Eine Evaluierung in bezug auf die Einfachheit der Bedienung, auf Usability Kriterien ist notwendig. An dieser Stelle muss festgestellt werden, dass die Erwartungshaltung der Nutzer in bezug auf die Einfachheit der Bedienung einer Website der öffentlichen Verwaltung sich nicht von der Erwartungshaltung in bezug auf eine E-Business Website unterscheidet. In der virtuellen Welt wollen User ihre (Navigations-) Erfahrungen und Kenntnisse anwenden, gleich ob sie eine E-Business oder VerwaltungsWebsite besuchen.

Eine klare, nutzerorientierte Informationsarchitektur ist das Basisgerüst für eine hervorragende Usability. Betreiber von Websites der öffentlichen Verwaltung sollten daher die informationsarchitektonischen Konzepte durchleuchten und gegebenenfalls adaptieren. Navigationskonzepte, die das Auffinden von bestimmten Inhalten und Services auf der Website erleichtern sollten in die Praxis umgesetzt werden, Konzepte, wie die Orientierung nach Lebenslagen oder nach Lebenssituationen sollten auch in die Praxis umgesetzt werden. 


\subsection{Informationsqualität beeinflusst nicht nur die Zufriedenheit sondern auch das Vertrauen in die Institution}

Dass die Qualität der angebotenen Informationen eine wichtige Rolle spielt, ist für die Websites der öffentlichen Verwaltung ebenso gültig, wie für Websites generell. Im besonderen konnte nachgewiesen werden, dass die Informationsqualität der Website nicht nur die Zufriedenheit mit der Website sondern auch das Vertrauen in die Institution beeinflusst. Daher wird Verwaltungspraktikern nahegelegt, die Inhalte von Websites zu evaluieren, da schlecht aufbereitete Seiten im Internet einen allgemeinen Vertrauensverlust der Institution bewirken können. Der inhaltlichen Qualitätsorientierung von Verwaltungs-Websites sollte ein hoher Stellenwert eingeräumt werden.

\section{References}

1. Amelingmeyer, Jenny: Wissensmanagement. Analyse und Gestaltung der Wissensbasis von Unternehmen. 2.Aufl. Wiesbaden 2002

2. Attems, R.; Holzer, A.: Spitzenleistungen in die Praxis umsetzen, Wien, 1989

3. BA, S.; Pavlou, P.A.: Evidence of the Effect of Trust Building Technology in Electronic Markets: Price Premiums and Buyer Behavior, MIS Quarterly, Vol.26, No.3, Sep. 2002, S.243-268

4. Backhaus, Klaus, Erichson, Bernd; Plinke Wulff; Weiber, Rolf: Multivariate Analysemethoden. Eine anwendungsorientierte Einführung, 10. Aufl. Springer; 2002

5. Bodendorf, Freimut: Daten- und Wissensmanagement. Springer 2003

6. Caldow, J.: The Quest for Electronic Government - A Defining Vision, Institute for Electronic Government, IBM Corporation, Washington, 1999, http://www.ieg.ibm.com/thought_leadership/egovvision.pdf (12.5.2003)

7. Chin, W.W.; Lee, M.: A Proposed Model for Measurement Instruments For The Formation of IS Satisfaction: The Case of End-User Computing Satisfaction, Proceedings of the Twenty First International Conference on Information Systems, 2000, S.553-563

8. Doll, W.J.; Torkzadeh, G.: A Discrepancy Model of End-User Computing Involvement, Management Science, Vol. 35, Oct. 1989, S.1151-1171

9. Doll, W.J.; Torkzadeh, G.: The Measurement of End-User Computing Satisfaction, MIS Quarterly, 12 (2), 1988, S.259-274

10. Downing, C.E.: System usage behavior as a proxy for user satisfaction: an empirical investigation, Information \& Management 35, 1999, S.203-216

11. Fogg, B.J; Kameda, T; Boyd, J., Marshall, J., Sethi, R., Sockol, M., Trowbrdige, T.: Stanford Makovsky Credibility Study 2002: Investigating what makes Web sites credible today, Research Report by the Stanford Persuasive Technology Lab \& Makovsky \& Company, 2002, S.1-16

12. Fogg, B.J; Marshall, J.; Laraki, O.; et al.: What makes Web Sites Credible?, Report on a large Quantitative Study, Persuasive Technology Lab-Chi-letters, Stanford University, 2001, S.61-68

13. Kaiser, H.F.; Rice, J.: Little Jiffy, Mark IV, in: Educational and Psychological Measurement, 34, 1974, S.111-117

14. Kaiser, K.M.; Srinivasan, A.: The Relationship of User Attitudes Toward Design Criteria and Information Systems Success, National AIDS Conference Proceedings, 1980, S. $203-210$

15. Kouufaris, M.; Hampton-Sosa, W.: Initial Perceptions of Company Trustworthiness Online: A Comprehensive Model and Empirical Test, CIS Working Paper Series, 2002, S.1-39 
16. Lucke, J. von: Portale für die öffentliche Verwaltung, Governmntal Portal, Departmental Portal und Life-Event Portal, in: Reinermann, H.; Lucke, J. von: Portale in der öffentlichen Verwaltung, Internet - Call Center - Bürgerbüro, Speyerer Forschungsbericht 205, Speyer, 2000, S.7-22

17. McHaney, R.; Hightower R., White, D.: EUCS Test-Reliability in Representational Model Decision Support Systems, Information \& Management, Vol. 36, No. 2, Aug. 1999, S.109-119

18. Reinermann, H.: Der öffentliche Sektor im Internet - Veränderungen der Muster öffentlicher Verwaltungen. Forschungsinstitut für öffentliche Verwaltung bei der Deutschen Hochschule für öffentliche Verwaltungswissenschaften, Speyerer Forschungsbericht Nr. 206, Speyer, 2000, S.11 ff.

19. Reinermann, H.: Portale - Tore des Wissens, in: Reinermann, H.; Lucke, J. von: Portale in der öffentlichen Verwaltung, Internet - Call Center - Bürgerbüro, Speyerer Forschungsbericht 205, Speyer, 2000

20. Pavlou, P. A. : Consumer Intentions to Adopt Electronic Commerce - Incorporating Trust and Risk in the Technology Acceptance Model , Paper to be presented in the DIGIT Workshop, 2001, S.1-28

21. Sultan, F.; Urban, G.L., Shankar, V., Bart, I.Y.: Determinants and Role of Trust in EBusiness: A Large Empirical Study, eBusiness Research center Working Paper, Dec. 2002, S.1-44

22. Wang, Y-S.; Tang, T-I. ; Tang, J. E. : An Instrument for Measuring Costumer Satisfaction Toward Web Sites that Market Digital Products and Services, Journal of Electronic Commerce Research, Vol. 2, No. 3, 2001, S.89-102 